\title{
S.A.W. TECHNOLOGY FOR RESONATOR STRUCTURES
}

\author{
F. SLEECKX, T. NATEN, A. VAN DE CAPELLE and J. VANDEWEGE $\dagger$ \\ K.U. Leuven, Department Elektrotechniek, E.S.A.T. Laboratory, Kardinaal Mercierlaan 94, \\ $B-3030$ Heverlee, Belgium
}

(Received August 30, 1979)

The different processing steps of S.A.W. technology are reviewed. Special attention is given to the technology of the distributed feedback resonator.

\section{INTRODUCTION}

Surface acoustic wave (S.A.W) structures have performed important functions as discrete components since the beginning of the decade. The earliest use was as filters in modern radar systems for pulse compression. Such filters are still the most common S.A.W. devices being used in systems today.

Surface acoustic waves are high-frequency sound waves travelling at the surface of a polished piezoelectric substrate. The substrates which are mostly applied, are ST-cut quartz, lithium niobate, lithium tantalate and bismuth germanium oxide. Each type of substrate has advantages and disadvantages. Lithium niobate is used for filters because of the large coupling coefficient $k^{2}$ of the substrate. ST-cut quartz substrates are used for oscillators which require a good temperature stability. Lithium niobate and ST-cut quartz are the two substrates which have been employed in our technology.

The S.A.W. technology discussed in this paper has been performed in relationship with the design of the distributed feedback resonator ${ }^{1,2}$ shown in Figure 1. This resonator structure has been realized as a metal pattern on a piezoelectric crystal.

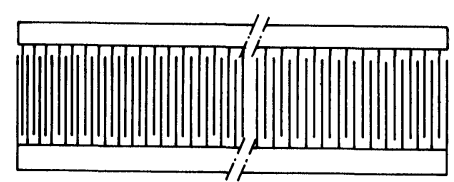

FIGURE 1 Distributed feedback resonator.

$\dagger$ Lab. for Electromagnetism and Acoustics, University of Ghent, St Pietersnieuwstraat, 41 - B-9000 Ghent, Belgium
In this case the S.A.W. resonator process is compatible with the highly developed manufacturing techniques used in the integrated circuit industry. The process consists of:

- mask generation

- deposition of metal layers on the substrate

- etching of patterns

- etching of grooves

- packaging

\section{MASK GENERATION}

The procedure of mask generation contains three steps:

- creation of the pattern on a computer by means of an easy to use computer program;

- cutting the stabilene with an off-line plotter;

- a photographic reduction of the stabilene mask.

The resonator structure of Figure 1 is a smallband structure. Such an interdigital structure requires a large number of fingers and spaces. With the facilities available at our laboratory, this number is limited by the off-line plotter and the photographic reduction step. The pattern on the stabilene has maximum dimensions of $0.86 \mathrm{~m} \times 0.78 \mathrm{~m}$. After the photographic reduction process the maximum dimensions are $38 \mathrm{~mm} \times 38 \mathrm{~mm}$.

The photo-reduction step can be performed on different kinds of masks. In this research program, a comparative study of emulsion, chromium and iron oxide masks has been made. From this study, it can be concluded that emulsion masks show a very wide transition zone between the black and white strips of the masks. This transition zone influences the finger-space ratio of the interdigital structure. 


\section{DEPOSITION OF THE METAL LAYERS ON THE SUBSTRATE}

The evaporation of the metal layers has been executed on a tungstene filament evaporation system. The evaporation of gold as well as aluminium has been examined. The adhesion of the metal layer on the piezoelectrical crystal is a critical point in this process, therefore chromium has been employed as an intermediate layer. It is worth mentioning the difficult adhesion of gold to piezoelectric substances. Analyzing different techniques, the best results are obtained with the following process: the chromium and gold layers are evaporated in one and the same evaporation process. Substrate heating during evaporation is a method of improving the adhesion. The resonator structures have been designed with a layer thickness of $3000 \AA$. In order to obtain a thickness between the tolerance limits of $\pm 10 \%$, the thickness has to be measured during deposition by means of a thickness monitor.

\section{ETCHING METHODS}

Three different methods have been studied.

\subsection{The Classical Etching Method}

Figure 2 gives an illustration of this technique.

The process consists of the following steps:

- cleaning of the substrate;

- deposition of the metal layers;

- covering the substrate with photoresist;

- exposure of the photoresist on a mask alignment system;

- a development step to remove either the exposed or unexposed polymer, thereby leaving the pattern in relief on the metal layer;

- etching the metal layers with hard etching products;

- removing of the resist.

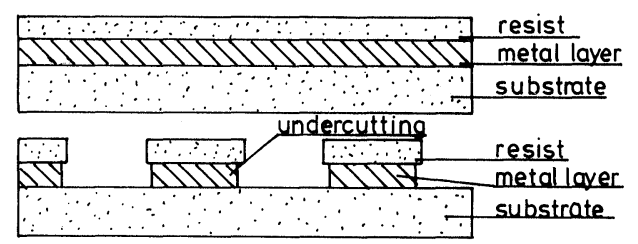

FIGURE 2 Chemical etching process - before exposure of the resist - after chemical etching
Concerning the realization of S.A.W. resonators with this technique the following points have to be compared.

Disadvantages:

1) The etching process causes an undercutting effect of the photoresist pattern (Figure 2).

2) The use of hard etching products, e.g. phosphoric acid, is necessary.

3) There is a need for very selective etching products for the different metals.

4) There are etching problems caused by the transition region between two different metal layers.

Advantages:

1) There are no special demands on the resist profile.

2) Substrate heating is possible.

3) Positive and negative resist can be used.

\subsection{Lift-off Technique}

The disadvantages of the classical etching method can be omitted by changing the sequence of the process steps, see Figure 3.

As a first step, the substrate is coated with a polymerfilm pattern. As a second step, the metal layer is deposited on this coated substrate. Dissolving the polymerfilm the metal will remain on the substrate as a negative pattern in comparison with the forementioned polymerfilm pattern.

A critical point in this process, concerns the transition between the metal deposited on the substrate surface and the metal on top of the polymer surface. If there exists a strong connection between both, "lift-off" will be very difficult without rather violent means e.g. ultrasonic vibration. In order to inhibit this effect, the side-walls of the resist profile have to be perpendicular or slightly undercut. During evaporation the metal has to be deposited at a nearly normal incidence angle.

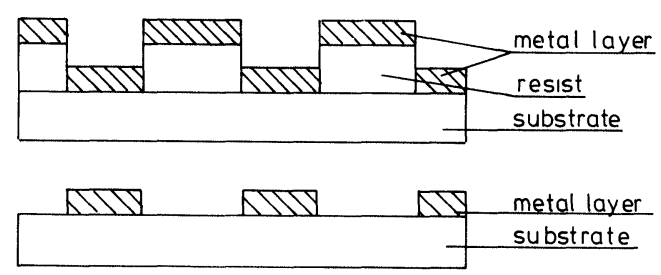

FIGURE 3 Lift-off technique - after deposition of the metal layers - after removing of the resist 
The required resist profile can be obtained with contact printing combined with conformable photomasks.

Applying the "lift-off" technique, adhesion between metal layer and substrate material cannot be improved by substrate heating during evaporation. Indeed, the photo-resist profile would not keep its sharp edges. Small traces of contamination degrade adhesion and have to be removed from the substrate surface before evaporation.

\subsection{R.F. Sputter Etching}

R.F. sputter etching (see Figure 4) is a variant of the classical chemical etching technology. The process does not use chemical etching products. Indeed, material will be removed by ion bombardment. This etching technique enables a high resolution of the finger-space ratio to be achieved and undercutting of the resist profile to be omitted.

Moreover, metal layers as well as substrate material can be etched in a very selective way, if the appropriate gas is available for each material.

The ion bombardment creates heat energy in the substrate. This energy has to be dissipated by means of a very good thermal contact between the substrate and the cathode of the sputter apparatus. Diffusion oil creates a good thermal contact.

This etching technique possesses the advantages of the chemical technique but it has the disadvantage of requiring a longer processing time.

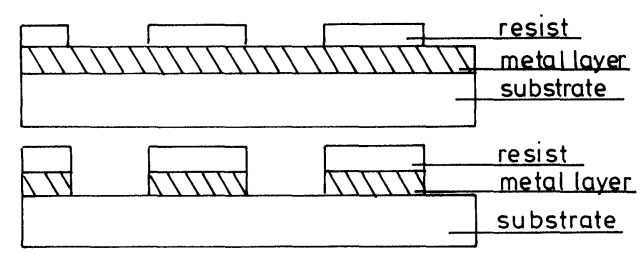

FIGURE 4 RF sputter etching technique

- after the development step of the resist

- after sputter etching

\subsection{Practical Realisation of Structures}

In our laboratory R.F. sputter etching has been combined with chemical etching. Chemical etching has been applied to obtain the pattern of the structure in the metal layer. Grooves have been sputtered in the substrate material between the fingers of the structure in order to increase the quality factor of the resonator (Figure 1). This method is preferred for resonator structures on quartz substrates.

For lithium niobate substrates, chemical etching as well as the lift-off techniques were successfully applied.

\section{PACKAGING}

After cutting of the substrate in the desired dimensions, it will be attached to a suitable package. The distributed feedback resonator structures have a great number of fingers. At V.H.F. frequencies, the length of the structure is such that a special package is needed.

A low value is required for the resonator serial resistance. Measurements have shown that special attention has to be paid to the bonding paths and to the diameter of the bonding wire. The interdigital transducer generates the desired acoustic surface waves but also undesired acoustic bulk waves. We obtained the best results when the other side of the substrate had been coarsened and when the substrate had been attached to the package with an appropriate bonding paste.

\section{CONCLUSION}

The different method for S.A.W. processing, especially for the distributed feedback resonator, have been presented. The proposed processes are compatible with the techniques applied in the integrated circuit technology.

\section{ACKNOWLEDGEMENTS}

This research was sponsored by N. V. Barco - Electronics through IWONL research project No. 2634, by IWONL research fellowships to T. Naten and J. Vandewege and by NFWO research fellowships to A Van de Capelle.

\section{REFERENCES}

1. J. Vandewege, P. E. Lagasse, T. Naten, F. Sleeckx, H. Tromp and G. Hoffman, "Acoustic surface wave resonators for broadband applications", Proc. 8th European Microwave Conference, Paris, 4 th -8 th September 1978, pp 663-667.

2. J. Vandewege, P E. Lagasse and T. Naten, "Distributed feedback acoustic surface wave resonators," Proc. I.E.E.E. Conference on Ultrasonics, September 1978, to be published. 

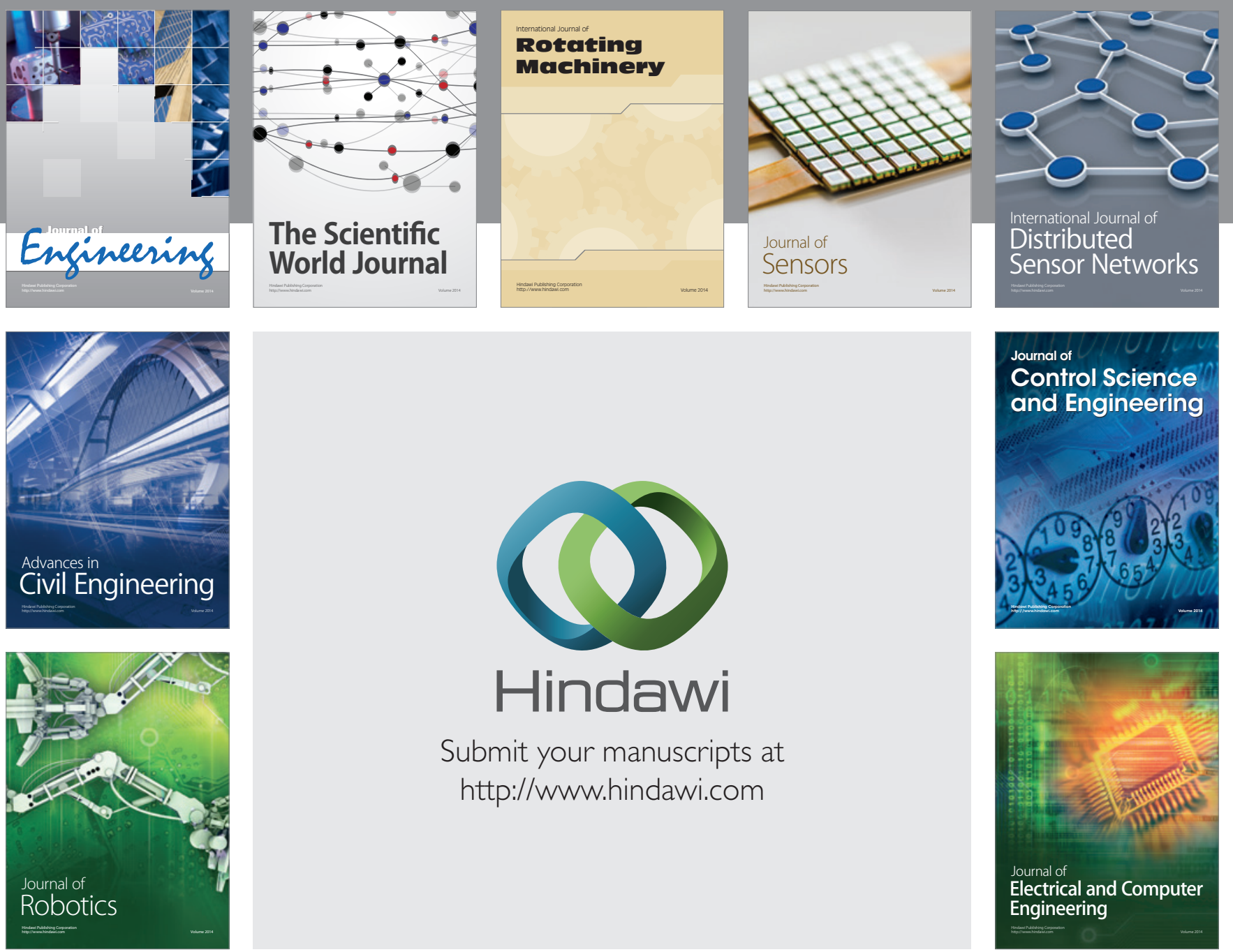

Submit your manuscripts at

http://www.hindawi.com
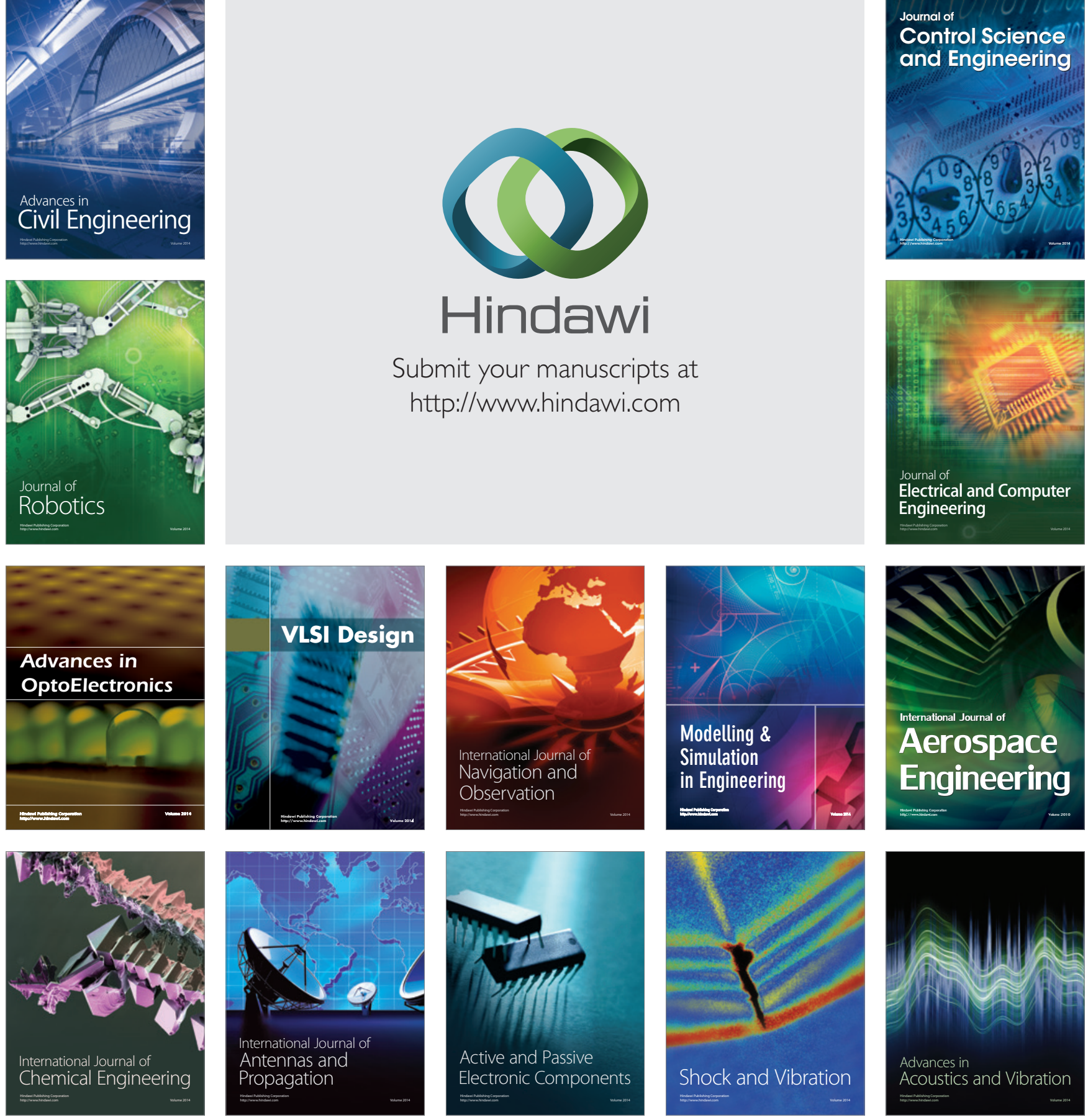